PUPT-1831

hep-th/9901018

\title{
From Threebranes to Large N Gauge Theories ${ }^{*}$
}

\author{
Igor R. Klebanov \\ Joseph Henry Laboratories \\ Princeton University \\ Princeton, New Jersey 08544, USA
}

\begin{abstract}
This is a brief introductory review of the AdS/CFT correspondence and of the ideas that led to its formulation. Emphasis is placed on dualities between conformal large $N$ gauge theories in 4 dimensions and string backgrounds of the form $A d S_{5} \times X_{5}$. Attempts to generalize this correspondence to asymptotically free theories are also included.
\end{abstract}

January 1999

${ }^{*}$ Based on the talk at Orbis Scientiae '98, Ft. Lauderdale, Florida, December 17-21, 1998. 


\section{Introduction}

It is well-known that string theory originated from attempts to understand the strong interactions [1]. However, after the emergence of QCD as the theory of hadrons, the dominant theme of string research shifted to the Planck scale domain of quantum gravity [2]. Although in hadron physics one routinely hears about flux tubes and the string tension, the majority of particle theorists gave up hope that string theory might lead to an exact description of the strong interactions. Now, however, for the first time we can say with confidence that at least some strongly coupled gauge theories have a dual description in terms of strings. Let me emphasize that one is not talking here about effective strings that give an approximate qualitative description, but rather about an exact duality. At weak coupling a convenient description of the theory involves conventional perturbative methods; at strong coupling, where such methods are intractable, the dual string description simplifies and gives exact information about the theory. The best established examples of this duality are conformal gauge theories where the so-called AdS/CFT correspondence [3, 4, 5] has allowed for many calculations at strong coupling to be performed with ease. In these notes I describe, from my own personal perspective, some of the ideas that led to the formulation of the AdS/CFT correspondence. I will also speculate on the future directions. For the sake of brevity I will mainly discuss the $\mathrm{AdS}_{5} / \mathrm{CFT}_{4}$ case which is most directly related to 4-dimensional gauge theories.

It has long been believed that the best hope for a string description of non-Abelian gauge theories lies in the 't Hooft large $N$ limit. A quarter of a century ago 't Hooft proposed to generalize the $S U(3)$ gauge group of QCD to $S U(N)$, and to take the large $N$ limit while keeping $g_{\mathrm{YM}}^{2} N$ fixed [6]. In this limit each Feynman graph carries a topological factor $N^{\chi}$, where $\chi$ is the Euler characteristic of the graph. Thus, the sum over graphs of a given topology can perhaps be thought of as a sum over world sheets of a hypothetical "QCD string." Since the spheres (string tree diagrams) are weighted by $N^{2}$, the tori (string one-loop diagrams) - by $N^{0}$, etc., we find that the closed string coupling constant is of order $N^{-1}$. Thus, the advantage of taking $N$ to be large is that we find a weakly coupled string theory. It is not clear, however, how to describe this string theory in elementary terms (by a 2-dimensional world sheet action, for example). This is clearly an important problem: the free closed string spectrum is just the large $N$ spectrum of glueballs. If the quarks are included, then we also find open strings describing the mesons. Thus, if methods are developed for calculating these spectra, and it is found that they are discrete, then this provides an elegant explanation of confinement. Furthermore, the $1 / N$ corrections correspond to perturbative string corrections.

Many years of effort, and many good ideas, were invested into the search for an 
exact gauge field/string duality [7]. One class of ideas, exploiting the similarity of the large $N$ loop equation with the string Schroedinger equation, eventually led to the following fascinating speculation [8]: one should not look for the QCD string in four dimensions, but rather in five, with the fifth dimension akin to the Liouville dimension of non-critical string theory [9]. This leads to a picture where the QCD string is described by a two-dimensional world sheet sigma model with a curved 5-dimensional target space. At that stage it was not clear, however, precisely what target spaces are relevant to gauge theories. Luckily, we now do have answers to this question for a variety of conformal large $N$ gauge models. The route that leads to this answer, and confirms the idea of the fifth dimension, involves an unexpected detour via black holes and Dirichlet branes. We turn to these subjects next.

\section{D-branes vs. Black Holes and $p$-branes}

A few years ago it became clear that, in addition to strings, superstring theory contains soliton-like "membranes" of various internal dimensionalities called Dirichlet branes (or D-branes) [10]. A Dirichlet $p$-brane (or D $p$-brane) is a $p+1$ dimensional hyperplane in $9+1$ dimensional space-time where strings are allowed to end, even in theories where all strings are closed in the bulk of space-time. In some ways a D-brane is like a topological defect: when a closed string touches it, it can open open up and turn into an open string whose ends are free to move along the D-brane. For the end-points of such a string the $p+1$ longitudinal coordinates satisfy the conventional free (Neumann) boundary conditions, while the $9-p$ coordinates transverse to the $\mathrm{D} p$-brane have the fixed (Dirichlet) boundary conditions; hence the origin of the term "Dirichlet brane." In a seminal paper [11] Polchinski showed that the D $p$-brane is a BPS saturated object which preserves $1 / 2$ of the bulk supersymmetries and carries an elementary unit of charge with respect to the $p+1$ form gauge potential from the Ramond-Ramond sector of type II superstring. The existence of BPS objects carrying such charges is required by non-perturbative string dualities [12]. A striking feature of the D-brane formalism is that it provides a concrete (and very simple) embedding of such objects into perturbative string theory.

Another fascinating feature of the D-branes is that they naturally realize gauge theories on their world volume. The massless spectrum of open strings living on a D pbrane is that of a maximally supersymmetric $U(1)$ gauge theory in $p+1$ dimensions. The $9-p$ massless scalar fields present in this supermultiplet are the expected Goldstone modes associated with the transverse oscillations of the $\mathrm{D} p$-brane, while the photons and fermions may be thought of as providing the unique supersymmetric completion. If we consider $N$ parallel D-branes, then there are $N^{2}$ different species of open strings because they can begin and end on any of the D-branes. $N^{2}$ is the dimension of the 
adjoint representation of $U(N)$, and indeed we find the maximally supersymmetric $U(N)$ gauge theory in this setting [13. The relative separations of the $\mathrm{D} p$-branes in the $9-p$ transverse dimensions are determined by the expectation values of the scalar fields. We will be primarily interested in the case where all scalar expectation values vanish, so that the $N \mathrm{D} p$-branes are stacked on top of each other. If $N$ is large, then this stack is a heavy object embedded into a theory of closed string which contains gravity. Naturally, this macroscopic object will curve space: it may be described by some classical metric and other background fields, such as the Ramond-Ramond $p+1$ form potential. Thus, we have two very different descriptions of the stack of D $p$-branes: one in terms of the $U(N)$ supersymmetric gauge theory on its world volume, and the other in terms of the classical Ramond-Ramond charged $p$-brane background of the type II closed superstring theory. It is the relation between these two descriptions that is at the heart of the recent progress in understanding the connections between gauge fields and strings.t Of course, more work is needed to make this relation precise.

\subsection{Counting the entropy}

The first success in building this kind of correspondence between black hole metrics and D-branes was achieved by Strominger and Vafa [15]. They considered 5dimensional supergravity obtained by compactifying 10-dimensional type IIB theory on a 5-dimensional compact manifold (for example, the 5-torus), and constructed a class of black holes carrying 2 separate $U(1)$ charges. These solutions may be viewed as generalizations of the well-known 4-dimensional charged (Reissner-Nordstrom) black hole. For the Reissner-Nordstrom black hole the mass is bounded from below by a quantity proportional to the charge. In general, when the mass saturates the lower (BPS) bound for a given choice of charges, then the black hole is called extremal. The extremal Strominger-Vafa black hole preserves 1/8 of the supersymmetries present in vacuum. Also, the black hole is constructed in such a way that, just as for the ReissnerNordstrom solution, the area of the horizon is non-vanishing at extremality [15]. In general, an important quantity characterizing black holes is the Bekenstein-Hawking entropy which is proportional to the horizon area:

$$
S_{B H}=\frac{A_{h}}{4 G}
$$

where $G$ is the Newton constant. Strominger and Vafa calculated the BekensteinHawking entropy of their extremal solution as a function of the charges and succeeded in reproducing this result with D-brane methods. To build a D-brane system carrying the same set of charges as the black hole, they had to consider intersecting D-branes

\footnotetext{
${ }^{1}$ There are other similar relations between large $N$ SYM theories and gravity stemming from the BFSS matrix theory conjecture 14.
} 
wrapped over the compact 5-dimensional manifold. For example, one may consider D3-branes intersecting over a line or D1-branes embedded inside D5-branes. The $1+1$ dimensional gauge theory describing such an intersection is quite complicated, but the degeneracy of the supersymmetric BPS states can nevertheless be calculated in the Dbrane description valid at weak coupling. For reasons that will become clear shortly, the description in terms of black hole metrics is valid only at very strong coupling. Luckily, due to the supersymmetry, the number of states does not change as the coupling is increased. This ability to extrapolate the D-brane counting to strong coupling makes a comparison with the Bekenstein-Hawking entropy possible, and exact agreement is found in the limit of large charges [15]. In this sense the collection of D-branes provides a "microscopic" explanation of the black hole entropy.

This correspondence was quickly generalized to black holes slightly excited above the extremality [16, 17]. Further, the Hawking radiation rates and the absorption crosssections were calculated and successfully reproduced by D-brane models [16, 18, 19]. Since then this system has been receiving a great deal of attention. However, some detailed comparisons are hampered by the complexities of the dynamics of intersecting D-branes: to date there is no first principles approach to the lagrangian of the $1+1$ dimensional conformal field theory on the intersection.

For this and other reasons it has turned out very fruitful to study a similar correspondence for simpler systems which involve parallel D-branes only [20, 21, 22, 23, 24]. Our primary motivation is that, as explained above, parallel $\mathrm{D} p$-branes realize $p+1$ dimensional $U(N)$ SYM theories, and we may learn something new about them from comparisons with Ramond-Ramond charged black p-brane classical solutions. These solutions in type II supergravity have been known since the early 90's [25, 26]. The metric and dilaton backgrounds may be expressed in the following simple and universal form:

$$
\begin{gathered}
d s^{2}=H^{-1 / 2}(r)\left[-f(r) d t^{2}+\sum_{i=1}^{p}\left(d x^{i}\right)^{2}\right]+H^{1 / 2}(r)\left[f^{-1}(r) d r^{2}+r^{2} d \Omega_{8-p}^{2}\right] \\
e^{\Phi}=H^{(3-p) / 4}(r)
\end{gathered}
$$

where

$$
H(r)=1+\frac{L^{7-p}}{r^{7-p}}, \quad f(r)=1-\frac{r_{0}^{7-p}}{r^{7-p}},
$$

and $d \Omega_{8-p}^{2}$ is the metric of a unit $8-p$ dimensional sphere. The horizon is located at $r=r_{0}$ and the extremality is achieved in the limit $r_{0} \rightarrow 0$. A solution with $r_{0} \ll L$ is called near-extremal. In contrast to the situation encountered for the StromingerVafa black hole, the Bekenstein-Hawking entropy vanishes in the extremal limit. Just like the stacks of parallel D-branes, the extremal solutions are BPS saturated: they preserve 16 of the 32 supersymmetries present in the type II theory. For $r_{0}>0$ the 
p-brane carries some excess energy $E$ above its extremal value, and the BekensteinHawking entropy is also non-vanishing. The Hawking temperature is then defined by $T^{-1}=\partial S_{B H} / \partial E$.

The correspondence between the entropies of the $p$-brane solutions (2) and those of the $p+1$ dimensional SYM theories was first considered in [20, 21]. Among these solutions $p=3$ has a special status: in the extremal limit $r_{0} \rightarrow 0$ the 3 -brane solution is perfectly non-singular [27]. This is evidenced by the fact that the dilaton $\Phi$ is constant for $p=3$, but blows up at $r=0$ for all other extremal solutions. In [20] the BekensteinHawking entropy of a near-extremal 3-brane of Hawking temperature $T$ was compared with the entropy of the $\mathcal{N}=4$ supersymmetric $U(N)$ gauge theory (which lives on $N$ coincident D3-branes) heated up to the same temperature. The results turned out to be quite interesting. The Bekenstein-Hawking entropy expressed in terms of the Hawking temperature $T$ and the number $N$ of elementary units of charge was found to be

$$
S_{B H}=\frac{\pi^{2}}{2} N^{2} V_{3} T^{3}
$$

where $V_{3}$ is the spatial volume of the 3-brane. This was compared with the entropy of a free $U(N) \mathcal{N}=4$ supermultiplet, which consists of the gauge field, $6 N^{2}$ massless scalars and $4 N^{2}$ Weyl fermions. This entropy was calculated using the standard statistical mechanics of a massless gas (the black body problem), and the answer turned out to be

$$
S_{0}=\frac{2 \pi^{2}}{3} N^{2} V_{3} T^{3}
$$

It is remarkable that the 3 -brane geometry captures the $T^{3}$ scaling characteristic of a conformal field theory (in a CFT this scaling is guaranteed by the extensivity of the entropy and the absence of dimensionful parameters). 2 Also, the $N^{2}$ scaling indicates the presence of $O\left(N^{2}\right)$ unconfined degrees of freedom, which is exactly what we expect in the $\mathcal{N}=4$ supersymmetric $U(N)$ gauge theory. On the other hand, the relative factor of $3 / 4$ between $S_{B H}$ and $S_{0}$ at first appeared mysterious and was interpreted by many as a subtle failure of the D3-brane approach to black 3-branes. As we will see shortly, however, the relative factor of $3 / 4$ is not a contradiction but rather a prediction about strongly coupled $\mathcal{N}=4$ SYM theory at finite temperature.

\footnotetext{
${ }^{2}$ Other examples of the "conformal" behavior of the Bekenstein-Hawking entropy include the 11dimensional 5-brane and membrane solutions [21]. For the 5-brane, $S_{B H} \sim N^{3} T^{5} V_{5}$, while for the membrane $S_{B H} \sim N^{3 / 2} T^{2} V_{2}$. The microscopic description of the 5-brane solution is in terms of a large number $N$ of coincident singly charged 5-branes of M-theory, whose chiral world volume theory has $(0,2)$ supersymmetry. Similarly, the membrane solution describes the large $N$ behavior of the CFT on $N$ coincident elementary membranes. The entropy formulae suggest that these theories have $O\left(N^{3}\right)$ and $O\left(N^{3 / 2}\right)$ massless degrees of freedom respectively. These predictions of supergravity 21] are non-trivial and still mysterious. Since the geometry of the 5-brane throat is $A d S_{7} \times S^{4}$, and that of the membrane throat is $A d S_{4} \times S^{7}$, these systems lead to other interesting examples of the AdS/CFT correspondence.
} 


\subsection{From absorption cross-sections to two-point correlators}

Almost a year after the entropy comparisons [20, 21] I came back to the 3-branes (and also to the 11-dimensional membranes and 5-branes) and tried to interpret absorption cross-sections for massless particles in terms of the world volume theories [22]. This was a natural step beyond the comparison of entropies, and for the Strominger-Vafa black holes the D-brane approach to absorption was initiated earlier in [16, 18]. For the system of $N$ coincident D3-branes it was interesting to inquire to what extent the supergravity and the weakly coupled D-brane calculations agreed. For example, they might scale differently with $N$ or with the incident energy. Even if the scaling exponents agreed, the overall normalizations could differ by a subtle numerical factor similar to the $3 / 4$ found for the 3 -brane entropy. Surprisingly, the low-energy absorption crosssections turned out to agree exactly!

To calculate the absorption cross-sections in the D-brane formalism one needs the low-energy world volume action for coincident D-branes coupled to the massless bulk fields. Luckily, these couplings may be deduced from the D-brane Born-Infeld action. For example, the coupling of 3-branes to the dilaton $\Phi$, the Ramond-Ramond scalar $C$, and the graviton $h_{\alpha \beta}$ is given by [22, 23]

$$
S_{\mathrm{int}}=\frac{\sqrt{\pi}}{\kappa} \int d^{4} x\left[\operatorname{tr}\left(\frac{1}{4} \Phi F_{\alpha \beta}^{2}-\frac{1}{4} C F_{\alpha \beta} \tilde{F}^{\alpha \beta}\right)+\frac{1}{2} h^{\alpha \beta} T_{\alpha \beta}\right],
$$

where $T_{\alpha \beta}$ is the stress-energy tensor of the $\mathcal{N}=4 \mathrm{SYM}$ theory. Consider, for instance, absorption of a dilaton incident on the 3-brane at right angles with a low energy $\omega$. Since the dilaton couples to $\operatorname{tr} F_{\alpha \beta}^{2}$ it can be converted into a pair of back-to-back gluons on the world volume. The leading order calculation of the cross-section for weak coupling gives [22]

$$
\sigma=\frac{\kappa^{2} \omega^{3} N^{2}}{32 \pi}
$$

where $\kappa=\sqrt{8 \pi G}$ is the 10-dimensional gravitational constant (note that the factor $N^{2}$ comes from the degeneracy of the final states which is the number of different gluon species). This result was compared with the absorption cross-section by the extremal 3-brane geometry,

$$
d s^{2}=\left(1+\frac{L^{4}}{r^{4}}\right)^{-1 / 2}\left(-d t^{2}+d x_{1}^{2}+d x_{2}^{2}+d x_{3}^{2}\right)+\left(1+\frac{L^{4}}{r^{4}}\right)^{1 / 2}\left(d r^{2}+r^{2} d \Omega_{5}^{2}\right) .
$$

This geometry may be viewed as a semi-infinite throat which for $r \gg L$ opens up into flat $9+1$ dimensional space. Waves incident from the $r \gg L$ region partly reflect back and partly penetrate into the the throat region $r \ll L$. The relevant s-wave radial equation turns out to be [22]

$$
\left[\frac{d^{2}}{d \rho^{2}}-\frac{15}{4 \rho^{2}}+1+\frac{(\omega L)^{4}}{\rho^{4}}\right] \psi(\rho)=0
$$


where $\rho=\omega r$. For a low energy $\omega \ll 1 / L$ we find a high barrier separating the two asymptotic regions. The low-energy behavior of the tunneling probability may be calculated by the so-called matching method, and the resulting absorption cross-section is 22]

$$
\sigma_{S U G R A}=\frac{\pi^{4}}{8} \omega^{3} L^{8}
$$

In order to compare (6) and (9) we need a relation between the radius of the throat, $L$, and the number of D3-branes, $N$. Such a relation follows from equating the ADM tension of the extremal 3-brane solution to $N$ times the tension of a single D3-brane, and one finds 20]

$$
L^{4}=\frac{\kappa}{2 \pi^{5 / 2}} N
$$

Substituting this into (9), we find that the supergravity absorption cross-section agrees exactly with the D-brane one, without any relative factor like $3 / 4$.

This result was a major surprise to me, and I started searching for its explanation. The most important question is: what is the range of validity of the two calculations? Since $\kappa \sim g_{s t} \alpha^{\prime 2}$, (10) gives $L^{4} \sim N g_{s t} \alpha^{\prime 2}$. Supergravity can only be trusted if the length scale of the 3 -brane solution is much larger than the string scale $\sqrt{\alpha^{\prime}}$, i.e. for $N g_{s t} \gg 1$. 0 Of course, the incident energy also has to be small compared to $1 / \sqrt{\alpha^{\prime}}$. Thus, the supergravity calculation should be valid in the "double-scaling limit" 22

$$
\frac{L^{4}}{\alpha^{\prime 2}} \sim g_{s t} N \rightarrow \infty, \quad \omega^{2} \alpha^{\prime} \rightarrow 0
$$

If the description of the black 3-brane by a stack of many coincident D3-branes is correct, and we presume that it is, then it must agree with the supergravity results in this limit. Since $g_{s t} \sim g_{\mathrm{YM}}^{2}$, this corresponds to the limit of infinite 't Hooft coupling in the $\mathcal{N}=4 U(N)$ SYM theory. Since we also want to send $g_{s t} \rightarrow 0$ in order to suppress the string loop corrections, we necessarily have to take the large $N$ limit. To summarize, the supergravity calculations are expected to give exact information about the $\mathcal{N}=4 \mathrm{SYM}$ theory in the limit of large $N$ and large 't Hooft coupling 22].

Coming back to the entropy problem, we now see that the Bekenstein-Hawking entropy calculation applies to the $g_{\mathrm{YM}}^{2} N \rightarrow \infty$ limit of the theory, while the free field calculation applies to the $g_{\mathrm{YM}}^{2} N \rightarrow 0$ limit. Thus, the relative factor of $3 / 4$ is not a discrepancy: it relates two different limits of the theory. Indeed, on general grounds we expect that in the 't Hooft large $N$ limit the entropy is given by 28

$$
S=\frac{2 \pi^{2}}{3} N^{2} f\left(g_{\mathrm{YM}}^{2} N\right) V_{3} T^{3} .
$$

\footnotetext{
${ }^{3}$ A similar conclusion applies to the Strominger-Vafa black hole [15].
} 
The function $f$ is certainly not constant: for example, a recent two-loop calculation 29] shows that its perturbative expansion is

$$
f\left(g_{\mathrm{YM}}^{2} N\right)=1-\frac{3}{2 \pi^{2}} g_{\mathrm{YM}}^{2} N+\ldots
$$

Thus, the Bekenstein-Hawking entropy in supergravity, (3), is translated into the prediction that $f\left(g_{\mathrm{YM}}^{2} N \rightarrow \infty\right)=3 / 4$. In fact, a recent string theory calculation of the leading strong coupling correction gives [28]

$$
f\left(g_{\mathrm{YM}}^{2} N\right)=\frac{3}{4}+\frac{45}{32} \zeta(3)\left(2 g_{\mathrm{YM}}^{2} N\right)^{-3 / 2}+\ldots .
$$

This is consistent with $f\left(g_{\mathrm{YM}}^{2} N\right)$ being a monotonic function which interpolates between 1 at $g_{\mathrm{YM}}^{2} N=0$ and $3 / 4$ at $g_{\mathrm{YM}}^{2} N=\infty$.

Although we have sharpened the region of applicability of the supergravity calculation (9), we have not yet explained why it agrees with the leading order perturbative result (6) on the D3-brane world volume. After including the higher-order SYM corrections, the general structure of the absorption cross-section in the large $N$ limit is expected to be 24

$$
\sigma=\frac{\kappa^{2} \omega^{3} N^{2}}{32 \pi} a\left(g_{\mathrm{YM}}^{2} N\right)
$$

where

$$
a\left(g_{\mathrm{YM}}^{2} N\right)=1+b_{1} g_{\mathrm{YM}}^{2} N+b_{2}\left(g_{\mathrm{YM}}^{2} N\right)^{2}+\ldots
$$

For agreement with supergravity, the strong 't Hooft coupling limit of $a\left(g_{\mathrm{YM}}^{2} N\right)$ should be equal to 1 [24]. In fact, a stronger result is true: all perturbative corrections vanish and $a=1$ independent of the coupling. This was first shown explicitly in [24] for the graviton absorption. The absorption cross-section is related to the imaginary part of the two-point function $\left\langle T_{\alpha \beta}(p) T_{\gamma \delta}(-p)\right\rangle$ in the SYM theory. In turn, this is determined by a conformal "central charge" which satisfies a non-renormalization theorem: it is completely independent of the "t Hooft coupling.

In general, the two-point function of a gauge invariant operator in the strongly coupled SYM theory may be read off from the absorption cross-section for the supergravity field which couples to this operator in the world volume action 24]. Some examples of this field operator correspondence may be read off from (5). Thus, we learn that the dilaton absorption cross-section measures the imaginary part of $\left\langle\operatorname{tr} F_{\alpha \beta}^{2}(p) \operatorname{tr} F_{\gamma \delta}^{2}(-p)\right\rangle$, the Ramond-Ramond scalar absorption cross-section measures the imaginary part of $\left\langle\operatorname{tr} F_{\alpha \beta} \tilde{F}^{\alpha \beta}(p) \operatorname{tr} F_{\gamma \delta} \tilde{F}^{\gamma \delta}(-p)\right\rangle$, etc. The agreement of these two-point functions with the weak-coupling calculations performed in [22, 23] is explained by supersymmetric non-renormalization theorems. Thus, the proposition that the $g_{\mathrm{YM}}^{2} N \rightarrow \infty$ limit of the large $N \mathcal{N}=4 \mathrm{SYM}$ theory can be extracted from the 3-brane of type IIB supergravity has passed its first consistency checks. 


\section{The AdS/CFT Correspondence}

The circle of ideas reviewed in the previous section received a seminal development by Maldacena [3] who also connected it for the first time with the QCD string idea. Maldacena made a simple and powerful observation that the "universal" region of the 3-brane geometry, which should be directly identified with the $\mathcal{N}=4$ SYM theory, is the throat, i.e. the region $r \ll L$. . The limiting form of the metric (7) is

$$
d s^{2}=\frac{L^{2}}{z^{2}}\left(-d t^{2}+d \vec{x}^{2}+d z^{2}\right)+L^{2} d \Omega_{5}^{2},
$$

where $z=\frac{L^{2}}{r} \gg L$. This metric describes the space $A d S_{5} \times S^{5}$ with equal radii of curvature $L$. One also finds that the self-dual 5 -form Ramond-Ramond field strength has constant flux through this space (the field strength term in the Einstein equation effectively gives a positive cosmological constant on $S^{5}$ and a negative one on $A d S_{5}$ ). Thus, Maldacena conjectured that type IIB string theory on $A d S_{5} \times S^{5}$ should be somehow dual to the large $N \mathcal{N}=4$ SYM theory.

Maldacena's argument was based on the fact that the low-energy $\left(\alpha^{\prime} \rightarrow 0\right)$ limit may be taken directly in the 3-brane geometry and is equivalent to the throat $(r \rightarrow 0)$ limit. Another way to motivate the identification of the gauge theory with the throat is to think about the absorption of massless particles considered in the previous section. In the D-brane description, a particle incident from the asymptotic infinity is converted into an excitation of the stack of D-branes, i.e. into an excitation of the gauge theory on the world volume. In the supergravity description, a particle incident from the asymptotic (large $r$ ) region tunnels into the $r \ll L$ region and produces an excitation of the throat. The fact that the two different descriptions of the absorption process give identical cross-sections supports the identification of excitations of $A d S_{5} \times S^{5}$ with the excited states of the $\mathcal{N}=4$ SYM theory.

Another strong piece of support for this identification comes from symmetry considerations [3]. The isometry group of $A d S_{5}$ is $S O(2,4)$, and this is also the conformal group in $3+1$ dimensions. In addition we have the isometries of $S^{5}$ which form $S U(4) \sim S O(6)$. This group is identical to the R-symmetry of the $\mathcal{N}=4$ SYM theory. After including the fermionic generators required by supersymmetry, the full isometry supergroup of the $A d S_{5} \times S^{5}$ background is $S U(2,2 \mid 4)$, which is identical to the $\mathcal{N}=4$ superconformal symmetry. We will see that in theories with reduced supersymmetry the compact $S^{5}$ factor becomes replaced by other compact spaces $X_{5}$, but $A d S_{5}$ is the "universal" factor present in the dual description of any large $N$ CFT and realizing the $S O(2,4)$ conformal symmetry. One may think of these backgrounds as type IIB theory compactified on $X_{5}$ down to 5 dimensions. Such Kaluza-Klein compactifications

\footnotetext{
${ }^{4}$ Related ideas were also pursued in 30 .
} 
of type IIB supergravity were extensively studied in the mid-eighties [31, 32, 33], and special attention was devoted to the $A d S_{5} \times S^{5}$ solution because it is a maximally supersymmetric background [34, 35]. It is remarkable that these early works on compactification of type IIB theory were actually solving large $N$ gauge theories without knowing it.

As Maldacena has emphasized, however, it is important to go beyond the supergravity limit and think of the $A d S_{5} \times X_{5}$ space as a background of string theory [3]. Indeed, type IIB strings are dual to the electric flux lines in the gauge theory, and this provides a natural set-up for calculating correlation functions of the Wilson loops. Furthermore, if $N$ is sent to infinity while $g_{\mathrm{YM}}^{2} N$ is held fixed and finite, then there are finite string scale corrections to the supergravity limit [3, 4, 5] which proceed in powers of

$$
\frac{\alpha^{\prime}}{L^{2}} \sim\left(g_{\mathrm{YM}}^{2} N\right)^{-1 / 2}
$$

If we wish to study finite $N$, then there are also string loop corrections in powers of

$$
\frac{\kappa^{2}}{L^{8}} \sim N^{-2}
$$

As expected, taking $N$ to infinity enables us to take the classical limit of the string theory on $A d S_{5} \times X_{5}$. However, in order to understand the large $N$ gauge theory with finite ' $t$ Hooft coupling, we should think of the $A d S_{5} \times X_{5}$ as the target space of a 2dimensional sigma model describing the classical string physics [4]. The fact that after the compactification on $X_{5}$ the string theory is 5-dimensional supports Polyakov's idea [8]. In $A d S_{5}$ the fifth dimension is related to the radial coordinate and, after a change of variables $z=L e^{-\varphi / L}$, the sigma model action turns into a special case of the general ansatz proposed in [8],

$$
I=\frac{1}{2} \int d^{2} \sigma\left[\left(\partial_{i} \varphi\right)^{2}+a^{2}(\varphi)\left(\partial_{i} X^{\mu}\right)^{2}+\ldots\right],
$$

where $a(\varphi)=e^{\varphi / L}$. It is clear, however, that the string sigma models dual to the gauge theories are of rather peculiar nature. The new feature revealed by the D-brane approach, which is also a major stumbling block, is the presence of the RamondRamond background fields. Little is known to date about such 2-dimensional field theories and, in spite of recent new insights [36, an explicit solution is not yet available.

\subsection{Correlation functions and the bulk/boundary correspon- dence}

Maldacena's work provided a crucial insight that the $A d S_{5} \times S^{5}$ throat is the part of the 3-brane geometry that is most directly related to the $\mathcal{N}=4$ SYM theory. 
It is important to go further, however, and explain precisely in what sense the two should be identified and how physical information can be extracted from this duality. Major strides towards answering these questions were made in two subsequent papers [4, 5] where essentially identical methods for calculating correlation functions of various operators in the gauge theory were proposed. As we mentioned in section 2.2, even prior to [3] some information about the field/operator correspondence and about the twopoint functions had been extracted from the absorption cross-sections. The reasoning

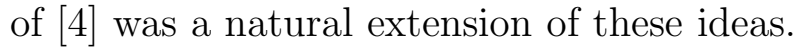

One may motivate the general method as follows. When a wave is absorbed, it tunnels from the asymptotic infinity into the throat region, and then continues to propagate toward smaller $r$. Let us separate the 3-brane geometry into two regions: $r \gtrsim L$ and $r \lesssim L$. For $r \lesssim L$ the metric is approximately that of $A d S_{5} \times S^{5}$, while for $r \gtrsim L$ it becomes very different and eventually approaches the flat metric. Signals coming in from large $r$ may be thought of as disturbing the "boundary" of $A d S_{5}$ at $r \sim L$, and then propagating into the bulk. This suggests that, if we discard the $r \gtrsim L$ part of the 3-brane metric, then we have to cut off the radial coordinate of $A d S_{5}$ at $r \sim L$, and the gauge theory correlation functions are related to the response of the string theory to boundary conditions. Guided by this idea, [4 proposed to identify the generating functional of connected correlation functions in the gauge theory with the extremum of the classical string action subject to the boundary conditions that $\phi\left(x^{\lambda}, z\right)=\phi_{b}\left(x^{\lambda}\right)$ at $z=L$ (at $z=\infty$ all fluctuations are required to vanish): :

$$
W\left[\phi_{b}\left(x^{\lambda}\right)\right]=S_{\phi_{b}\left(x^{\lambda}\right)}
$$

$W$ generates the connected Green's functions of the gauge theory operator that corresponds to the field $\phi$ in the sense explained in section 2.2, while $S_{\phi_{b}\left(x^{\lambda}\right)}$ is the extremum of the classical string action subject to the boundary conditions. An essentially identical prescription was also proposed in [5] with a somewhat different motivation. If we are interested in the correlation functions at infinite ' $t$ Hooft coupling, then the problem of extremizing the classical string action reduces to solving the equations of motion in type IIB supergravity whose form is known explicitly [34]. Note that from the point of view of the metric (16) the boundary conditions are imposed not at $z=0$ (which would be a true boundary of $A d S_{5}$ ) but at some finite value $z=z_{\text {cutoff }}$. It does not matter which value it is since it can be changed by an overall rescaling of the coordinates $\left(z, x^{\lambda}\right)$ which leaves the metric unchanged. The physical meaning of this cut-off is that it acts as a UV cut-off in the gauge theory [4, 37]. Indeed, the radial coordinate of $A d S_{5}$ is to be thought of as the effective energy scale of the gauge theory [3], and decreasing $z$ corresponds to increasing energy. In some calculations one may

${ }^{5}$ As usual, in calculating the correlation functions in a CFT it is convenient to carry out the Euclidean continuation. On the string theory side we then have to use the Euclidean version of $A d S_{5}$. 
remove the cut-off from the beginning and specify the boundary conditions at $z=0$, but in others the cut-off is needed at intermediate stages and may be removed only at the end 38 .

There is a growing literature on explicit calculations of correlation functions following the proposal of [4, 5]. In these notes we will limit ourselves to a brief discussion of the 2-point functions. Their calculations show that the dimensions of gauge invariant operators are determined by the masses of the corresponding fields in $A d S_{5}$ 《4, 5]. For scalar operators this relation is

$$
\Delta=2+\sqrt{4+(m L)^{2}} .
$$

Therefore, the operators in the $\mathcal{N}=4$ large $N$ SYM theory naturally break up into two classes: those that correspond to the Kaluza-Klein states of supergravity and those that correspond to massive string states. Since the radius of the $S^{5}$ is $L$, the masses of the Kaluza-Klein states are proportional to $1 / L$. Thus, the dimensions of the corresponding operators are independent of $L$ and therefore independent of $g_{\mathrm{YM}}^{2} N$. On the gauge theory side this is explained by the fact that the supersymmetry protects the dimensions of certain operators from being renormalized: they are completely determined by the representation under the superconformal symmetry [39, 40]. All families of the Kaluza-Klein states, which correspond to such BPS protected operators, were classified long ago 35 .

On the other hand, the masses of string excitations are $m^{2}=\frac{4 n}{\alpha^{\prime}}$ where $n$ is an integer. For the corresponding operators the formula (21) predicts that the dimensions do depend on the ' $t$ Hooft coupling and, in fact, blow up for large $g_{\mathrm{YM}}^{2} N$ as $2\left(n g_{\mathrm{YM}} \sqrt{2 N}\right)^{1 / 2}$. This is a highly non-trivial prediction of the AdS/CFT duality which has not yet been verified on the gauge theory side.

It is often stated that the gauge theory lives on the boundary of $A d S_{5}$. A more precise statement is that the gauge theory corresponds to the entire $A d S_{5}$, with the effective energy scale measured by the radial coordinate. In this correspondence the bare (UV) quantities in the gauge theory are indeed specified at the boundary of $A d S_{5}$. In calculating the correlation functions it is crucial that the boundary values of various fields in $A d S_{5}$ act as the sources in the gauge theory action which couple to gauge invariant operators as in (5). A similar connection arises in the calculation of Wilson loop expectation values [41. A Wilson loop is specified by a contour in $x^{\lambda}$ space placed at $z=z_{\text {cutoff }}$. One then looks for a minimal area surface in $A d S_{5}$ bounded by this contour and evaluates the Nambu action $I_{0}$ which is proportional to the area. The semiclassical value of the Wilson loop is then $e^{-I_{0}}$. This prescription, which is motivated by the duality between fundamental strings and electric flux lines, gives interesting results which are consistent with the conformal invariance [41]. For example, the quark-antiquark potential scales as $\sqrt{g_{\mathrm{YM}}^{2} N} /|\vec{x}|$. Note that this strong 
coupling result is different from the weak coupling limit where we have $V \sim g_{\mathrm{YM}}^{2} N /|\vec{x}|$.

\subsection{Conformal field theories and Einstein manifolds}

As we mentioned above, the duality between strings on $A d S_{5} \times S^{5}$ and the $\mathcal{N}=4$ SYM is naturally generalized to dualities between strings on $A d S_{5} \times X_{5}$ and other conformal gauge theories. The 5 -dimensional compact space $X_{5}$ is required to be a postively curved Einstein manifold, i.e. one for which $R_{\mu \nu}=\Lambda g_{\mu \nu}$ with $\Lambda>0$. The number of supersymmetries in the dual gauge theory is determined by the number of Killing spinors on $X_{5}$.

The simplest examples of $X_{5}$ are the orbifolds $S^{5} / \Gamma$ where $\Gamma$ is a discrete subgroup of $S O(6)$ 42, 43]. In these cases $X_{5}$ has the local geometry of a 5-sphere. The dual gauge theory is the IR limit of the world volume theory on a stack of $N$ D3-branes placed at the orbifold singularity of $R^{6} / \Gamma$. Such theories typically involve product gauge groups $S U(N)^{k}$ coupled to matter in bifundamental representations 44 .

Constructions of the dual gauge theories for Einstein manifolds $X_{5}$ which are not locally equivalent to $S^{5}$ are also possible. The simplest example is the Romans compactification on $X_{5}=T^{1,1}=(S U(2) \times S U(2)) / U(1)$ [32, 45]. It turns out that the dual gauge theory is the conformal limit of the world volume theory on a stack of $N$ D3-branes placed at the singularity of a certain Calabi-Yau manifold known as the conifold. This turns out to be the $\mathcal{N}=1$ superconformal field theory with gauge group $S U(N) \times S U(N)$ coupled to two chiral superfields in the $(\mathbf{N}, \overline{\mathbf{N}})$ representation and two chiral superfields in the $(\overline{\mathbf{N}}, \mathbf{N})$ representation [45]. This theory has an exactly marginal quartic superpotential which produces a critical line related to the radius of $A d S_{5} \times T^{1,1}$.

\section{Towards Non-conformal Gauge Theories in Four Dimensions}

In the preceding sections I hope to have convinced the reader that type IIB strings on $A d S_{5} \times X_{5}$ shed genuinely new light on four-dimensional conformal gauge theories. While many insights have already been achieved, I am convinced that a great deal remains to be learned in this domain. We should not forget, however, that the prize question is whether this duality can be extended to QCD or at least to other gauge theories which exhibit the asymptotic freedom and confinement. It is immediately clear that this will not be easy because, as we remarked in section 3, a string approach to weakly coupled gauge theory has not yet been fully developed (the well-understood supergravity limit describes gauge theory with very large ' $t$ Hooft coupling). On the 
other hand, the asymptotic freedom makes the coupling approach zero in the UV region [46]. Nevertheless, there may be some at least qualitative approaches to non-conformal gauge theories that shed light on the essential physical phenomena.

One such approach, proposed by Witten [47, builds on the observation that thermal gauge theories are described by near-extremal $p$-brane solutions [20, 21]. It is also known that the high temperature limit of a supersymmetric gauge theory in $p+1$ dimensions is described by non-supersymmetric gauge theory in $p$ dimensions. Thus, 3-dimensional non-supersymmetric gauge theory is dual to the throat region of the near-extremal 3-brane solution which turns out to have the geometry of a black hole in $A d S_{5}$ [47 (similar black holes were studied long ago by Hawking and Page 48]). Similarly, 4-dimensional non-supersymmetric gauge theory is dual to the near-horizon region of the near-extremal 4-brane solution [47. Witten calculated the Wilson loop expectation values in these geometries and showed that they satisfy the area law. Furthermore, calculations of the glueball masses produce discrete spectra with strong resemblance to the lattice simulations [49]. Unfortunately, this supergravity model has some undesirable features as well: for example, the presence in the geometry of a large $8-p$ dimensional sphere introduces into the spectrum families of light "KaluzaKlein glueballs" which are certainly absent from the lattice results. Presumably, the root of the problems is that the bare " $t$ Hooft coupling is taken to be large, while in order to achieve the conventional continuum limit it has to be sent to zero along a renormalization group trajectory.

A pessimistic conclusion would be that little more can be done at present because the supergravity approximation is supposed to be poor at weak 't Hooft coupling. Nevertheless, I feel that one should not give up attempts to understand the asymptotic freedom on the string side of the duality. In fact, some progress in this direction was recently achieved in [50, 51, 52 following Polyakov's suggestion [53] on how to break supersymmetry. Polyakov argued that a string dual of non-supersymmetric gauge theory should have world sheet supersymmetry without space-time supersymmetry. Examples of such theories include the type 0 strings, which are NSR strings with a non-chiral GSO projection which breaks the space-time supersymmetry [54.

There are two type 0 theories, $\mathrm{A}$ and $\mathrm{B}$, and both have no space-time fermions in their spectra but produce modular invariant partition functions [54]. The massless bosonic fields are as in the corresponding type II theory (A or B), but with the doubled set of the Ramond-Ramond (R-R) fields. The type 0 theory also contains a tachyon, which is why it has not received much attention thus far. In [53, 50 it was suggested, however, that the presence of the tachyon does not spoil its application to large $N$ gauge theories. A well-established route towards gauge theory is via the D-branes which were first considered in the type 0 context in 55. Large $N$ gauge theories, which are constructed on $N$ coincident D-branes of type 0 theory, may be shown to 
contain no open string tachyons [50, 53].

In [50] the presence of a bulk tachyon was turned into an advantage because it gives rise to the renormalization group flow. There the $3+1$ dimensional $S U(N)$ theory coupled to 6 adjoint massless scalars was constructed as the low-energy description of $N$ coincident electric D3-branes of type 0B theory.9 The conjectured dual type 0 background thus carries $N$ units of electric 5-form flux. The dilaton decouples from the $\left(F_{5}\right)^{2}$ terms in the effective action, and the only source for it originates from the tachyon mass term,

$$
\nabla^{2} \Phi=\frac{1}{8} m^{2} e^{\frac{1}{2} \Phi} T^{2}, \quad m^{2}=-\frac{2}{\alpha^{\prime}} .
$$

Thus, the tachyon background induces a radial variation of $\Phi$. Since the radial coordinate is related to the energy scale of the gauge theory, the effective coupling decreases toward the ultraviolet. In [51, 52 the UV limit of the type 0B background dual to the gauge theory was studied in more detail and a solution was found where the geometry is $A d S_{5} \times S^{5}$ while the "t Hooft coupling flows logarithmically. A calculation of the quark-antiquark potential showed qualitative agreement with what is expected in an asymptotically free theory.

These results raise the hope that the AdS/CFT duality can indeed be generalized to asymptotically free gauge theories. While we are still far from constructing reliable string duals of such theories, the availability of new ideas on this old and difficult problem makes me hopeful that more surprises lie ahead.

\section{Acknowledgements}

I am grateful to S. Gubser, A. Peet, A. Polyakov, A. Tseytlin and E. Witten, my collaborators on parts of the material reviewed in these notes. I also thank B. Kursunoglu and other organizers of Orbis Scientiae '98, especially L. Dolan (the convener of the string session), for sponsoring a very interesting conference. This work was supported in part by the NSF grant PHY-9802484 and by the James S. McDonnell Foundation Grant No. 91-48.

\section{References}

[1] Y. Nambu, "Quark model and the factorization of the Veneziano amplitude," in Symmetries and Quark Models, ed. R. Chand, Gordon and Breach (1970); H.

\footnotetext{
${ }^{6}$ In the type $0 \mathrm{~B}$ theory the 5 -form R-R field strength $F_{5}$ is not constrained to be selfdual. Therefore, it is possible to have electrically or magnetically charged 3-branes. This should be contrasted with the situation in the type IIB theory where the 5-form strength is constrained to be selfdual and, thus, only the selfdual 3-branes are allowed.
} 
B. Nielsen, "An almost physical interpretation of the integrand of the n-point Veneziano amplitude," submitted to the 15th International Conference on High Energy Physics, Kiev (1970); L. Susskind, "Dual-symmetric theory of hadrons," Nuovo Cim. 69A (1970) 457.

[2] J. Scherk and J. Schwarz, "Dual models for non-hadrons," Nucl. Phys. B81 (1974) 118.

[3] J. Maldacena, "The Large N limit of superconformal field theories and supergravity," Adv. Theor. Math. Phys. 2 (1998) 231, hep-th/9711200.

[4] S.S. Gubser, I.R. Klebanov, and A.M. Polyakov, "Gauge theory correlators from noncritical string theory," Phys. Lett. B428 (1998) 105, hep-th/9802109.

[5] E. Witten, "Anti-de Sitter space and holography," Adv. Theor. Math. Phys. 2 (1998) 253, hep-th/9802150.

[6] G. 't Hooft, "A planar diagram theory for strong interactions," Nucl. Phys. B72 (1974) 461.

[7] See, for example, A.M. Polyakov, "Gauge Fields and Strings," Harwood Academic Publishers (1987).

[8] A.M. Polyakov, "String theory and quark confinement," Nucl. Phys. B (Proc. Suppl.) 68 (1998) 1, hep-th/9711002.

[9] A.M. Polyakov, "Quantum geometry of bosonic strings," Phys. Lett. B103 (1981) 207.

[10] For a review, see J. Polchinski, "TASI lectures on D-branes," hep-th/9611050.

[11] J. Polchinski, "Dirichlet branes and Ramond-Ramond charges," Phys. Rev. Lett. 75 (1995) 4724, hep-th/9510017.

[12] C.M. Hull and P.K. Townsend, "Unity of superstring dualities," Nucl. Phys. B438 (1995) 109; P.K. Townsend, "The eleven-dimensional supermembrane revisited," Phys. Lett. B350 (1995) 184; E. Witten, "String theory dynamics in various dimensions," Nucl. Phys. B443 (1995) 85.

[13] E. Witten, "Bound states of strings and p-branes," Nucl. Phys. B460 (1996) 335, hep-th/9510135.

[14] T. Banks, W. Fischler, S. Shenker and L. Susskind, "M Theory as a matrix model: a conjecture," Phys. Rev. D55 (1997) 5112, hep-th/9610043.

[15] A. Strominger and C. Vafa, "Microscopic origin of the Bekenstein-Hawking entropy," Phys. Lett. B379 (1996) 99 , hep-th/9601029. 
[16] C.G. Callan and J.M. Maldacena, "D-brane approach to black hole quantum mechanics," Nucl. Phys. B472 (1996) 591, hep-th/9602043.

[17] G. Horowitz and A. Strominger, "Counting states of near-extremal black holes," Phys. Rev. Lett. 77 (1996) 2368, hep-th/9602051.

[18] A. Dhar, G. Mandal and S. Wadia, "Absorption vs. decay of black holes in string theory and T symmetry," Phys. Lett. B388 (1996) 51; S. Das and S. Mathur, "Comparing decay rates for black holes and D-branes," Nucl. Phys. B478 (1996) 561.

[19] J.M. Maldacena and A. Strominger, "Black hole grey body factors and D-brane spectroscopy," Phys. Rev. D55 (1997) 861.

[20] S.S. Gubser, I.R. Klebanov, and A.W. Peet, "Entropy and temperature of black 3-branes," Phys. Rev. D54 (1996) 3915, hep-th/9602135; A. Strominger, unpublished.

[21] I.R. Klebanov and A.A. Tseytlin, "Entropy of near-extremal black p-branes," Nucl. Phys. B475 (1996) 164, hep-th/9604089.

[22] I.R. Klebanov, "World volume approach to absorption by nondilatonic branes," Nucl. Phys. B496 (1997) 231, hep-th/9702076.

[23] S.S. Gubser, I.R. Klebanov, and A.A. Tseytlin, "String theory and classical absorption by three-branes," Nucl. Phys. B499 (1997) 217, hep-th/9703040.

[24] S.S. Gubser and I.R. Klebanov, "Absorption by branes and Schwinger terms in the world volume theory," Phys. Lett. B413 (1997) 41, hep-th/9708005.

[25] G.T. Horowitz and A. Strominger, "Black strings and p-branes," Nucl. Phys. B360 (1991) 197.

[26] M.J. Duff and J.X. Lu, "The selfdual type IIB superthreebrane," Phys. Lett. B273 (1991) 409; M.J. Duff, R. Khuri, J.X. Lu, "String solitons," Phys. Rept. 259 (1995) 213.

[27] G.W. Gibbons and P.K. Townsend, "Vacuum interpolation in supergravity via super p-branes," Phys. Rev. Lett. 71 (1993) 3754, hep-th/9307049.

[28] S.S. Gubser, I.R. Klebanov, and A.A. Tseytlin, "Coupling constant dependence in the thermodynamics of $\mathrm{N}=4$ supersymmetric Yang-Mills theory," Nucl. Phys. B534 (1998) 202, hep-th/9805156.

[29] A. Fotopoulos, T.R. Taylor, "Comment on two loop free energy in $N=4$ supersymmetric Yang-Mills theory at finite temperature," hep-th/9811224. 
[30] S. Hyun, "U-duality between Three and Higher Dimensional Black Holes," hep-th/9704005;

K. Sfetsos and K. Skenderis, "Microscopic derivation of the Bekenstein-Hawking entropy formula for non-extremal black holes," hep-th/9711138.

[31] M. Günaydin, L.J. Romans and N. Warner, "Compact and non-compact gauged supergravity in five dimensions," Nucl. Phys. B272 (1986) 598.

[32] L. Romans, "New compactifications of chiral $N=2, d=10$ supergravity," Phys. Lett. B153 (1985) 392.

[33] M.J. Duff, B.E.W. Nilsson and C.N. Pope, "Kaluza-Klein supergravity," Phys. Rep. 130 (1986) 1.

[34] J.H. Schwarz, "Covariant field equations of chiral $N=2, D=10$ supergravity," Nucl. Phys. B226 (1983) 269.

[35] H.J. Kim, L.J. Romans and P. van Nieuwenhuizen, "Mass spectrum of chiral ten dimensional $\mathcal{N}=2$ supergravity on $\mathbf{S}^{5}$," Phys. Rev. D32 (1985) 389; M. Günaydin and N. Marcus, "The spectrum of the $S^{5}$ compactification of the chiral $N=2, D=$ 10 supergravity and the unitary supermultiplets of $U(2,2 \mid 4)$," Class. Quant. Grav. 2 (1985) L11.

[36] R.R. Metsaev and A.A. Tseytlin, "Type IIB superstring action in $A d S_{5} \times S^{5}$ background," Nucl. Phys. B533 (1998) 109, hep-th/9805028; R. Kallosh and A.A. Tseytlin, "Simplifying superstring action on $A d S_{5} \times S^{5}$," J. High Energy Phys. 9810 (1988) 016, hep-th/9808088.

[37] L. Susskind and E. Witten, "The holographic bound in Anti-de Sitter space," hep-th/9805114.

[38] D.Z. Freedman, S. Mathur, A. Matusis and L. Rastelli, "Correlation functions in the $\mathrm{CFT}_{D} / \mathrm{AdS}_{D+1}$ correspondence," hep-th/9804058.

[39] P.S. Howe and P.C. West, "Superconformal invariants and extended supersymmetry," Phys. Lett. B400 (1997) 307.

[40] S. Ferrara, C. Fronsdal and A. Zaffaroni, "On $N=8$ supergravity on $A d S_{5}$ and $N=4$ superconformal Yang-Mills theory," Nucl. Phys. B532 (1998) 153, hep-th/9802203.

[41] J. Maldacena, "Wilson loops in large $N$ field theories," Phys. Rev. Lett. 80 (1998) 4859, hep-th/9803002; S.-J. Rey and J. Yee, "Macroscopic strings as heavy quarks in large $N$ gauge theory and anti-de Sitter supergravity", hep-th/9803001.

[42] S. Kachru and E. Silverstein, "4d conformal field theories and strings on orbifolds," hep-th/9802183. 
[43] A. Lawrence, N. Nekrasov and C. Vafa, "On conformal field theories in four dimensions," hep-th/9803015.

[44] M.R. Douglas and G. Moore, "D-branes, quivers, and ALE instantons," hep-th/9603167.

[45] I.R. Klebanov and E. Witten, "Superconformal field theory on threebranes at a Calabi-Yau singularity," Nucl. Phys. B536 (1998) 199, hep-th/9807080.

[46] D.J. Gross and F. Wilczek, "Ultraviolet behavior of nonabelian gauge theories," Phys. Rev. Lett. 30 (1973) 1343; H.D. Politzer, "Reliable perturbative results for strong interactions?" Phys. Rev. Lett. 30 (1973) 1346.

[47] E. Witten, "Anti-de Sitter space, thermal phase transition, and confinement in gauge theories," Adv. Theor. Math. Phys. 2 (1998) 505, hep-th/9803131.

[48] S.W. Hawking and D. Page, "Thermodynamics of black holes in anti de Sitter space," Commun. Math. Phys. 87 (1983) 577.

[49] C. Csaki, H. Ooguri, Y. Oz and J. Terning, "Glueball mass spectrum from supergravity," hep-th/9806021; R. De Mello Koch, A. Jevicki, M. Mihailescu and J. Nunes, "Evaluation of glueball masses from supergravity," hep-th/9806125; A. Hashimoto and Y. Oz, "Aspects of QCD dynamics from string theory," hep-th/9809106.

[50] I. R. Klebanov and A. A. Tseytlin, "D-Branes and dual gauge theories in type 0 strings," hep-th/9811035.

[51] J. Minahan, "Glueball mass spectra and other issues for supergravity duals of QCD models," hep-th/9811156.

[52] I. R. Klebanov and A. A. Tseytlin, "Asymptotic freedom and infrared behavior in the type 0 string approach to gauge theory," hep-th/9812089.

[53] A.M. Polyakov, "The wall of the cave," hep-th/9809057.

[54] L. Dixon and J. Harvey, "String theories in ten dimensions without space-time supersymmetry", Nucl. Phys. B274 (1986) 93; N. Seiberg and E. Witten, "Spin structures in string theory", Nucl. Phys. B276 (1986) 272; C. Thorn, unpublished.

[55] O. Bergman and M. Gaberdiel, "A non-supersymmetric open string theory and S-Duality," Nucl. Phys. B499 (1997) 183, hep-th/9701137. 